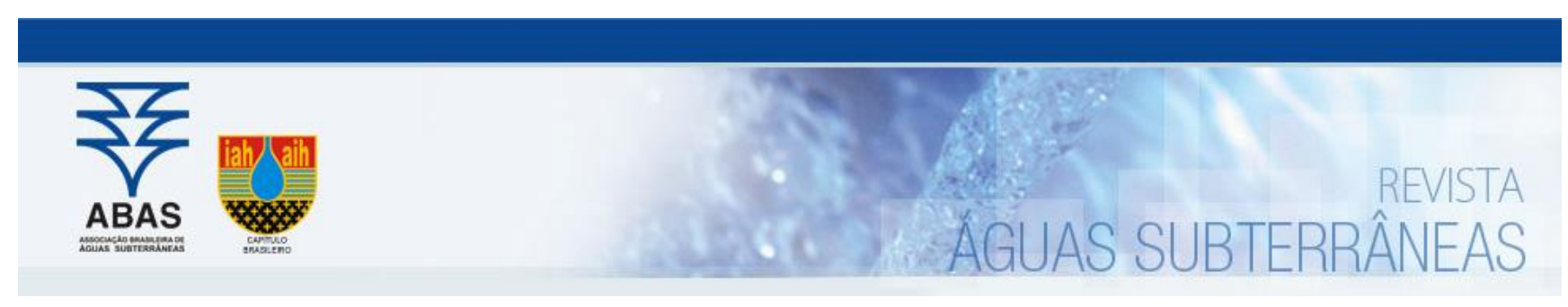

Artigos

\title{
Determinação do fator de retardamento da uranina em sedimentos quaternários do aquífero São Paulo
}

\section{Determination of the retardation factor of uranine in quaternary sediments of the São Paulo aquifer}

\author{
Otávio Barbosa Ferreira ${ }^{1}$; Alexandra Vieira Suhogusoff1; Tatiana Luiz dos Santos Tavares ${ }^{\circledR}$ \\ 1 Instituto de Geociências da Universidade de São Paulo (IGc-USP), São Paulo, SP, Brasil. \\ 2 Instituto de Pesquisas Tecnológicas do Estado de São Paulo (IPT), São Paulo, SP, Brasil.
}

$\bowtie$ otavio.barbosa.ferreira@usp.br, suhogusoff@usp.br, ttavares@ipt.br

Palavras-chave:

Traçador fluorescente.

Uranina.

Fator de retardamento.

$\underline{\text { Resumo }}$

A aplicação de substâncias fluorescentes como traçadores hidrogeológicos pode fornecer informações relevantes acerca da dinâmica das águas subterrâneas. Para um melhor controle na aplicação dos traçadores in situ, é pertinente definir possíveis interações dessas substâncias nos solos e sedimentos através de experimentos de bancada, a fim de prever seu comportamento durante a injeção no aquífero. 0 objetivo do trabalho corresponde à quantificação da adsorção e determinação do fator de retardamento do traçador fluorescente uranina em sedimentos quaternários da porção rasa do aquífero São Paulo. Os coeficientes de partição água/sedimento para uranina foram obtidos por meio de ensaios do tipo batch-test. Os experimentos consistiram em misturar sedimento orgânico, argiloso e grosso (arenoso) com soluções contendo uranina em cinco concentrações iniciais (5, 10, 50, 100 e 150 $\mathrm{\mu g} / \mathrm{L}$ ) em água deionizada e água do aquífero. Etapas de agitação, repouso e centrifugação precederam as medições de fluorescência. Através do ajuste de isotermas lineares, os valores do coeficiente de partição e fator de retardamento calculados para o sedimento grosso foram, respectivamente: $2,60 \times 10-3 \mathrm{~L} / \mathrm{g} \pm 0,22 \times 10-3$ e 1,51 $\pm 0,13$ (água

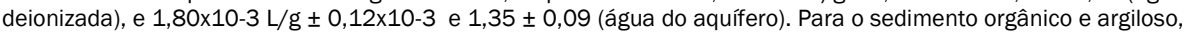
a fluorescência aparente gerada pelas partículas em suspensão nas soluções contendo uranina se sobrepôs à fluorescência real do traçador, impossibilitando a aferição dos coeficientes de partição e dos fatores de retardamento. Em meio sólido poroso com alto conteúdo de matéria orgânica e argilominerais, a uranina exibe alta susceptibilidade aos fenômenos de sorção. Contudo, sua utilização é indicada para a aferição de propriedades hidráulicas em aquíferos francamente quartzosos, uma vez nestes sedimentos a uranina apresentou um baixo fator de retardamento.

Keywords:

Abstract

Fluorescent tracer.

Uranine.

The application of fluorescent substances as hydrogeological tracers may provide relevant information about subsurface water dynamics. For a better in situ control in the application of tracers, it is relevant to define the chemical and physical behavior of these substances in soils and sediments through bench laboratory experiments in order to predict their behavior during the injection into the aquifer. The objective of this work was to quantify the adsorption and the retardation factor of the fluorescence tracer uranine in quaternary sediments of the upper portion of the São Paulo aquifer. The water/soil distribution coefficients for uranine were obtained by means of batch-tests assays. The experiments consisted in mixing three different soils (organic, clayey and coarse) with uranine solutions in five initial concentrations $(5,10,50,100$ and $150 \mu \mathrm{g} / \mathrm{L})$. The fluorescence measurements of the liquid phase were performed through a laboratory fluorometer. The calculated values of the distribution coefficient and retardation factor for the coarse soil were, respectively: $2,60 \times 10-3 \mathrm{~L} / \mathrm{g} \pm 0,22 \times 10-3$ and 1,51 $\pm 0,13$ (deionized water), and 1,80x10-3 L/g \pm $0,12 \times 10-3$ and $1,35 \pm 0,09$ (aquifer water). For organic and clayey soils, the apparent fluorescence generated by the suspended particles in the uranine solutions overlapped the real fluorescence of the tracer, preventing the distribution coefficient and the retardation factor from being calculated. In a porous medium with a high content of organic matter and clay minerals, uranine exhibits high susceptibility to sorption phenomena. However, its use is indicated for the measurement of hydraulic properties in quartz-rich aquifers, since in this sediments the uranine presented a low retardation factor.

DOI: http:/dx.doi.org/10.14295/ras.v35i1.29951

\section{INTRODUÇÃO}

As técnicas de aplicação de traçadores em estudos hidrogeológicos auxiliam na caracterização do comportamento das águas subterrâneas. Os traçadores podem ser importantes ferramentas de aprimoramento de modelos conceituais sobretudo de aquíferos hidrogeologicamente complexos, nos quais o meio físico pode apresentar condutividade hidráulica anisotrópica, 
porosidade secundária ou descontinuidades estratigráficas (CAO et al., 2020; DIVINE e MCDONNELL, 2005; SILVA et al., 2009).

Através de medições diretas ou indiretas de suas concentrações, a injeção de traçadores pode revelar características hidráulicas e geométricas do aquífero, como caminhos preferenciais de fluxo, dispersão hidrodinâmica, velocidade advectiva e zonas de maior ou menor condutividade hidráulica. Mais recentemente, essas substâncias também vêm sendo utilizadas como traçadores de poluentes orgânicos em aquíferos (BOTTRELL et al., 2010; PONSIN et al., 2015).

Estudos acerca dos mecanismos de controle de adsorção de uranina em sedimentos ainda são incompletos e pouco sistematizados (BORK et al., 2020). Portanto, a avaliação de possíveis interações físicas entre o traçador e o material sólido pelo qual ele percolará deve, preferencialmente, preceder sua apli- cação no aquífero, de modo a antever possíveis perdas de massa.

0 traçador fluorescente selecionado para esse estudo foi a uranina, também denominada de fluoresceína sódica ou amarelo ácido 73. Sua escolha foi embasada em características como elevada intensidade de fluorescência (IF), baixo limite de detecção, fácil manipulação e armazenamento. Os sedimentos foram amostrados da porção rasa do aquífero São Paulo, o qual é alvo de projetos de investigação ambiental devido à presença de inúmeros focos de contaminação em suas águas.

\section{2. ÁREA DE ESTUDO}

A área de estudo está localizada na zona oeste do município de São Paulo (SP) sobre os sedimentos aluvionares holocêni$\cos$ da bacia do rio Pinheiros (Figura 1).

Figura 1 - Mapa geológico do município de São Paulo com a localização da área de estudo

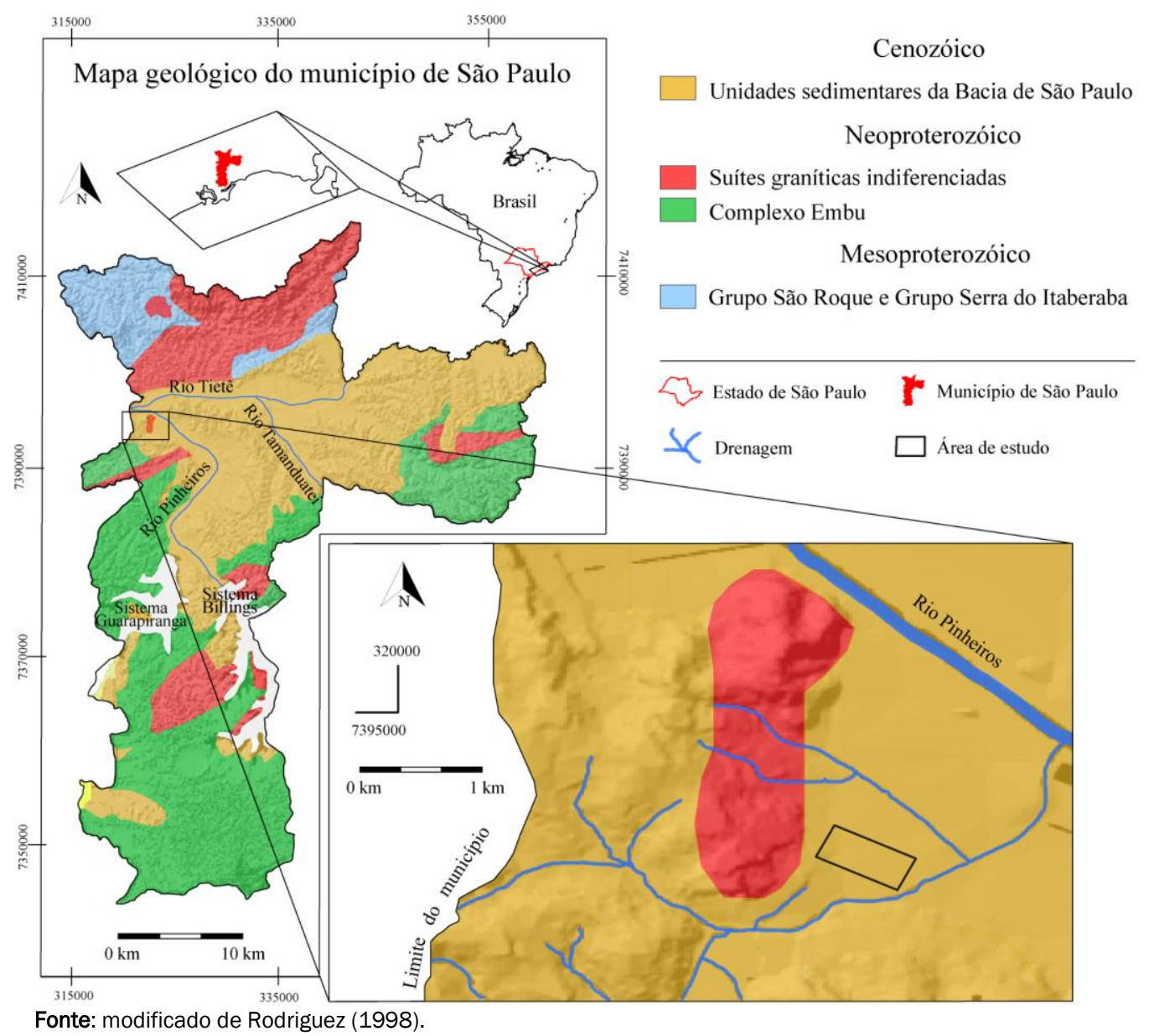

O arcabouço geológico do município de São Paulo insere-se no contexto do Rift Continental do Sudeste do Brasil (RICCOMINI, 1990). Sobre o embasamento pré-cambriano, assentam-se os sedimentos cenozoicos da Bacia de São Paulo, a qual compreende um hemi-graben basculado para NNW (RICCOMINI et al., 2004).
O embasamento pré-cambriano é definido pelos metamorfitos do Complexo Embu e dos grupos São Roque e Serra do Itaberaba (HASUl et al., 1975). O arcabouço litológico do embasamento também é formado por diversas intrusões graníticas neoproterozoicas indiferenciadas (JANASI e ULBRICH, 1991; JULIANI, 1993). Nessas rochas, desenvolve-se o aquífero Pré- 
Cambriano, aflorante na porção leste do Estado de São Paulo (FERNANDES et al., 2007).

A sucessão estratigráfica da Bacia de São Paulo é definida por rochas sedimentares terrígenas oligocênicas a miocênicas das formações Resende, Tremembé, São Paulo, Itaquaquecetuba e depósitos colúvio-aluvionares quaternários que recobrem todas as demais unidades (RICCOMINI, 1990; RODRIGUEZ, 1998; TAKIYA, 1997). O aquífero São Paulo é formado pelas associações sedimentares da Bacia de São Paulo e compreende uma área de aproximadamente $1.000 \mathrm{~km}^{2}$ na porção central da bacia hidrográfica do alto curso do rio Tietê (ROCHA, 2005).

As unidades hidroestratigráficas que ocorrem na área de estudo são compostas por depósitos sedimentares quaternários. Localmente, o aquífero possui geometria livre a semiconfinada, com intercalações centimétricas entre camadas arenosas e argilosiltosa. A profundidade do nível d'água situa-se entre 5,0 m e 6,5 m, com variações decorrentes de corpos d'água suspensos sobre a superfície freática principal, definidos em sua base por leitos predominantemente pelíticos associados à dinâmica deposicional fluvial em ambiente de leito de inundação sazonal (IPT, 2017).

O fluxo de água subterrânea possui sentido preferencial para sudeste, influenciado por dois córregos que margeiam a área a leste. A descarga regional do aquífero ocorre no rio Pinheiros, localizado a aproximadamente $1,5 \mathrm{~km}$ a nordeste da área (IPT, 2017).

\section{TRAÇADORES EM HIDROGEOLOGIA}

Em estudos hidrogeológicos, duas categorias de traçadores podem ser definidas, a depender da maneira como foram inseridos na área de estudo: naturais ou artificiais (KASS, 1998). Os traçadores naturais estão presentes no sistema como parte do contexto hidrogeológico da região, podendo ainda ser ambientais ou antropogênicos (LEIBUNDGUT et al., 2009). Traçadores artificiais são aqueles introduzidos proposital e controladamente no aquífero com o objetivo de compreender os comportamentos de fluxo e transporte da água subterrânea através de monitoramento (LEIBUNDGUT et al., 2009).

Segundo Ribeiro (2007), substâncias fluorescentes são comumente utilizadas como traçadores artificiais em estudos hidrológicos. Tratam-se de compostos orgânicos que apresentam fluorescência, ou seja, são capazes de absorver energia em diferentes comprimentos de onda e retransmiti-la, instantaneamente, sob a forma espectral de luz visível. Esse fenômeno físico ocorre devido à emissão de energia na forma de fótons (IUz) por parte de alguns elétrons ao retornarem às suas posições menos energéticas (LEIBUNDGUT e SEIBERT, 2011).

Comparados aos traçadores artificiais não fluorescentes, como os sais cloreto de sódio $(\mathrm{NaCl})$, cloreto de potássio $(\mathrm{KCl})$, cloreto de lítio ( $\mathrm{LiCl})$, borax $\left(\mathrm{Na}_{2} \mathrm{~B}_{4} \mathrm{O}_{7}\right)$ e brometo de sódio $(\mathrm{NaBr})$, os traçadores fluorescentes destacam-se devido ao baixo limite de detecção, na ordem de $\mu \mathrm{g} / \mathrm{L}$ (ppb) (LEIBUNDGUT e SEIBERT, 2011).

Há uma grande variedade de corantes fluorescentes disponíveis para compor soluções experimentais com diferentes intensidades de fluorescência e características físico-químicas. Contudo, as tinturas xanthene são preferíveis como traçadores hidrogeológicos por serem: (i) solúveis em água; (ii) facilmente detectáveis; (iii) fluorescentes em uma faixa do espectro incomum para compostos encontrados na água; (iv) atóxicas em baixas concentrações e (v) razoavelmente estáveis sob condições ambientais normais (WILSON JR et al., 1986).

Algumas características físicas e químicas dos traçadores fluorescentes mais utilizados em estudos hidrológicos encontram-se na Tabela 1.

Tabela 1 - Características físico-químicas dos principais traçadores fluorescentes.

\begin{tabular}{|c|c|c|c|c|c|}
\hline Traçador & Grupo & Fórmula química & $\begin{array}{l}\text { Limite de } \\
\text { detecção } \\
\left(\mathrm{mg} / \mathrm{m}^{3}\right)^{a}\end{array}$ & $\begin{array}{c}\text { Excitação/ } \\
\text { emissão } \\
(n m)^{a}\end{array}$ & $\begin{array}{c}\text { Solubilidade } \\
\text { em água } \\
(\mathrm{g} / \mathrm{L})\left(20^{\circ} \mathrm{C}\right) \text { a }\end{array}$ \\
\hline Uranina & & $\mathrm{C}_{20} \mathrm{H}_{10} \mathrm{O}_{5} \mathrm{Na}_{2}$ & 0,001 & $491 / 516$ & 300 \\
\hline \multirow[t]{2}{*}{ Rodamina WT } & & $\mathrm{C}_{29} \mathrm{H}_{29} \mathrm{O}_{5} \mathrm{ClN}_{2} \mathrm{Na}_{2}$ & 0,02 & $561 / 586$ & $3-20$ \\
\hline & Xanthene & & & & \\
\hline Rodamina B & & $\mathrm{C}_{28} \mathrm{H}_{31} \mathrm{O}_{3} \mathrm{ClN}_{2}$ & 0,02 & $555 / 575$ & $3-20$ \\
\hline Eosina & & $\mathrm{C}_{20} \mathrm{H}_{6} \mathrm{O}_{5} \mathrm{Br}_{4} \mathrm{Na}_{2}$ & 0,01 & $515 / 540$ & 300 \\
\hline
\end{tabular}

a Fonte: Leibundgut et al. (2009).

\subsection{Uranina}

A toxicidade dos principais traçadores fluorescentes utilizados em hidrogeologia foi analisada por FIELD et al. (1995) através do método SAR (Structure Activity Relationship). Os estudos revelaram que a uranina não apresenta comportamento troficamente cumulativo e seus riscos carcinogênicos, oncogênicos e mutagenéticos são baixíssimos quando utilizados em concentrações abaixo de $2 \mathrm{mg} / \mathrm{L}$ em permanência máxima de 24 horas. 0 risco toxicológico e ecotoxicológico do traçador foi 
definido, respectivamente, como baixo e moderado (FIELD et al., 1995; GOMBERT et al., 2017).

A fluorescência de fundo em uma solução está diretamente relacionada à presença de sedimentos em suspensão e matéria orgânica (coloidal e dissolvida) na água (TRUDGILL, 1987). Smart e Laidlaw (1977) reportaram uma correlação linear entre a concentração de carbono orgânico em água natural e sua fluorescência no intervalo de emissão entre 400-600 nm, sendo que diversas substâncias orgânicas apresentam pico de emissão entre 450-500 nm. Partículas em suspensão aumentaram a fluorescência de fundo aparente das soluções e reduziram a fluorescência efetiva dos corantes devido à absorção e espalhamento de luz pelos sedimentos. 0 espectro de emissão da matéria orgânica em águas naturais pode se sobrepor ao da uranina, cujo pico é de $516 \mathrm{~nm}$, enviesando os resultados obtidos pelo fluorímetro. Portanto, o uso dessa substância deve vir acompanhado de medições de fluorescência de fundo para definir o intervalo ideal das concentrações do traçador a ser utilizado.

A uranina apresenta uma das maiores taxas de decaimento fotoquímico entre as tinturas xanthene (SMART e LAIDLAW, 1977). A exposição à luz (LEIBUNDGUT e SEIBERT, 2011) tem um efeito irreversível sobre a fluorescência, portanto a dependência fotolítica do traçador deve ser levada em consideração durante a sua manipulação.

Mudanças nos valores de pH são capazes de deslocar a carga elétrica da molécula de negativa para neutra a positiva e viceversa, alterando o espectro de excitação e emissão do traçador fluorescente (LEIBUNDGUT e SEIBERT, 2011). Smart e Laidlaw (1977) e Suhogusoff et al. (2005) discutiram o comportamento de corantes fluorescentes, incluindo a uranina. Os autores relataram forte dependência da intensidade de fluorescência do traçador em função do $\mathrm{pH}$, a qual diminui exponencialmente em soluções ácidas.

A sorção dos corantes fluorescentes está associada às questões de conservatividade, dispersão e retardação, principalmente quando a solução apresenta material sólido em suspensão. A mudança no potencial hidrogeniônico da solução altera a carga elétrica superficial do traçador e, consequentemente, controlar sua taxa de sorção. A presença de fases orgânicas no sedimento, como serragem e húmus, resulta em uma maior taxa de adsorção de uranina comparado a fases inorgânicas, como quartzo, argilas e materiais carbonáticos, chegando a perdas de até $100 \%$ em massa do traçador em solução (SABATINI e AUSTIN, 1991).

\section{BATCH-TESTS}

Os batch-tests são ensaios de laboratório que permitem determinar a massa sorvida de um determinado soluto (Equação 1) em um material sólido. 0 termo sorção refere-se ao processo de transferência de massa entre o soluto dissolvido na água e o soluto presente no meio físico poroso (ZHENG, 1990), no qual se assume que uma concentração de equilíbrio entre as fases é atingida.
Através da determinação do coeficiente de partição entre o sólido e a água (Equação 2), estima-se, por meio da Equação 3 , o fator de retardamento $(R)$.

Estes ensaios consistem em montar frascos com uma solução do soluto de interesse dissolvido em água $\left(\mathrm{V}_{\text {solução}}\right)$ em contato com uma massa do material do aquífero ( $\left.m_{\text {sedimentos}}\right)$. Posteriormente, as misturas são submetidas a uma etapa de agitação antes de permanecerem em repouso, para que atinjam as concentrações de equilíbrio.

Após o sistema atingir o equilíbrio, é medida a concentração final na solução (C) para se estimar a massa de soluto sorvida no meio sólido (S). São realizados experimentos para diversas concentrações iniciais $\left(\mathrm{C}_{0}\right)$ a fim de se construir uma curva que relacione fase adsorvida (S) e fase dissolvida $(C)$. O coeficiente de partição corresponde ao coeficiente angular do ajuste entre $\mathrm{C}$ e S, considerando a isoterma linear.

Para o intervalo de concentrações na ordem de $\mu \mathrm{g} / \mathrm{L}$, o ajuste realizado por meio de isotermas lineares descreve de maneira satisfatória o comportamento do adsorvato devido às baixas concentrações, assumindo a suficiência no número de sítios sorptivos presentes na massa de sedimentos utilizada.

$$
\begin{aligned}
& S=\frac{\left(C_{0}-C\right) * V_{\text {soluşo }}}{m_{\text {sedimento }}} \\
& K_{d}=\frac{S}{C} \\
& R=1+\left(\rho / n_{e}\right) * K_{d}
\end{aligned}
$$

Onde,

S: Concentração adsorvida [M/M]

$\mathrm{C}_{0}$ : Concentração inicial da solução [M/L3]

C: Concentração final da solução [M/L3]

Vsolução: Volume da solução [L3]

Msedimentos: Massa de sedimentos [M]

$\mathrm{K}_{\mathrm{d}}$ : Coeficiente de adsorção [L3/M]

R: Fator de retardamento []

$\rho_{b}$ : Densidade seca do sedimento [M/L3]

$\mathrm{n}_{\mathrm{e}}$ : Porosidade específica []

\section{MATERIAIS E MÉTODOS}

\subsection{Coleta e caracterização física dos sedimentos}

Os ensaios de laboratório com uranina foram realizados com os diferentes tipos de sedimentos coletados na porção rasa do aquífero São Paulo, a até aproximadamente $9 \mathrm{~m}$ de profundidades, com amostradores do tipo liner de $44 \mathrm{~mm}$ de diâmetro, através de sondagem percussiva com perfuratriz GeoHammer®.

A caracterização granulométrica e composicional dos sedi- 
mentos foi definida através de descrições tácteis-visuais baseadas na coloração, aspereza, plasticidade, dispersão em água e resistência a seco dos materiais. Foi utilizada a difratometria de raios $\mathrm{X}$ para definir a mineralogia e o teor da fração argila nos sedimentos (Tabela 2). A análise composicional das argilas foi baseada no Método da Fração Argilosa para materiais menores que $0,002 \mathrm{~mm}$ em lâmina orientada. As amostras foram analisadas por um difratômetro Panalytical modelo Empyrean ${ }^{\circledR}$ sob três condições: (i) natural, (ii) glicolada e (iii) calcinada a $490^{\circ} \mathrm{C}$.
Através da integração dos dados físicos das amostras, definiram-se três sedimentos de granulação e composição distintas: (i) orgânico: argila siltosa preta rica em matéria orgânica; (ii) argiloso: argila plástica cinza-escura; (iii) grosso (arenoso): areia quartzosa média a grossa, cinza-clara com lentes centimétricas de cascalho.

Por uma questão de sistematização, os sedimentos orgânico e argiloso foram designados de finos ao longo deste trabalho.

Tabela 2 - Teor dos argilominerais presentes nos sedimentos finos

\begin{tabular}{ccc}
\hline \multirow{2}{*}{ Sedimento } & Grupo do argilomineral & $\begin{array}{c}\text { Teor } \\
\text { (\%) }\end{array}$ \\
\hline \multirow{2}{*}{ Orgânico } & Caulinita & $95-96$ \\
& Vermiculita & $1-2$ \\
& Illita & $2-3$ \\
\hline \multirow{3}{*}{ Argiloso } & Caulinita & $80-85$ \\
& Vermiculita & $5-10$ \\
& piolita & $2-3$ \\
& Illita & $<2$ \\
\hline
\end{tabular}

\subsection{Preparo das amostras de sedimentos}

Para os ensaios de tempo de agitação, equilíbrio químico e batch-tests, foram utilizadas porções de $10 \mathrm{~g}$ de sedimentos para cada solução contendo uranina.

O preparo das amostras teve início com a secagem de $2 \mathrm{~kg}$ de cada tipo de sedimento. Foi utilizada uma estufa de circulação e renovação de ar Solab $S L 102 \AA$ a $48^{\circ} \mathrm{C}$. 0 sedimento grosso permaneceu por 6 horas e os finos permaneceram por 40 horas na estufa até a secagem completa. Um moinho de panela Contenco I-4227 ${ }^{\circledR}$ foi utilizado para a desagregação dos sedimentos finos após a secagem.

As amostras foram quarteadas e individualizadas em porções de $10 \mathrm{~g}$ com o uso de uma balança de precisão Shimadzu AW200 ${ }^{\circledR}$.

\subsection{Medição de uranina}

Os ensaios foram realizados com um fluorímetro Turner TD$700 \AA$, equipado com o kit óptico para uranina. 0 kit é composto por uma lâmpada de vapor de mercúrio e filtros de excitação e emissão de $486 \mathrm{~nm}$ e $510-700 \mathrm{~nm}$, respectivamente. 0 método de calibração utilizado foi o Multi-point for Direct Concentrations (TURNER DESIGNS, 2002) para 5 concentra-

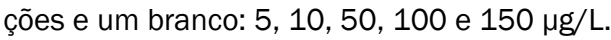

Antes de cada leitura realizada pelo fluorímetro durante todo o ensaio, as amostras foram induzidas ao pH 10 por adição de solução de hidróxido de sódio $(\mathrm{NaOH})$ em concentração normal (40 g/L). A alcalinização das amostras foi realizada para que a maior intensidade de fluorescência da uranina fosse atingida. As leituras de $\mathrm{pH}$ foram acompanhadas por um pHmetro Digimed DM22 ${ }^{\circledR}$.

Foram preparadas duas soluções-padrão para a calibração do fluorímetro, uma com água deionizada e outra com água do aquífero. A água deionizada foi utilizada como controle para os experimentos, pois não apresenta espécies químicas interferentes que podem eventualmente ser encontradas em águas naturais. Em cada solução-padrão foi diluído $10 \mathrm{mg}$ de uranina em $1 \mathrm{~L}$ de água. Elas foram armazenadas em balões volumétricos abrigados da luz.

O laboratório de análise foi mantido a uma temperatura constante de $22^{\circ} \mathrm{C}\left( \pm 1{ }^{\circ} \mathrm{C}\right)$ ao longo dos ensaios. Os frascos e recipientes de laboratório passaram por processos de lavagem antes e após cada utilização.

\subsection{Ensaios de aferição do comportamento químico da ura- nina}

Os ensaios de caracterização do comportamento químico da uranina forneceram resultados quantitativos acerca das variáveis tempo de agitação, tempo de equilíbrio e pH, as quais definem condições de controle para a realização dos batchtests. Todas as soluções foram manipuladas em frascos âmbar para evitar possíveis efeitos do decaimento fotoquímico da uranina.

\section{i) Tempo de agitação}

O ensaio de tempo de agitação foi realizado para caracterizar a relação entre o tempo de interação dinâmica sedimento/uranina e a concentração final de uranina na solução.

Foram montadas 3 soluções de $100 \mathrm{~mL}$ com água deionizada e uranina $(75 \mu \mathrm{g} / \mathrm{L})$ para os 3 tipos de sedimento. As amostras foram montadas em duplicatas. As soluções com cada sedimento foram agitadas em uma mesa agitadora horizontal para os intervalos de tempo de 30, 120 e 240 min a 33 rpm. Após a agitação, as amostras foram submetidas a um descanso de 24 horas.

Antes das medições realizadas pelo fluorímetro, as amostras foram centrifugadas por $30 \mathrm{~min}$ a $2250 \mathrm{rpm}$, para que ocor- 
resse a decantação de partículas suspensas.

\section{ii) Tempo de equilíbrio químico}

Foram realizados ensaios para definir o tempo de equilíbrio entre os sedimentos e a uranina. Em duplicata, foram montadas 5 soluções de $100 \mathrm{ml}$ de uranina em água deionizada (75 $\mu \mathrm{g} / \mathrm{L}$ ) para $10 \mathrm{~g}$ de cada sedimento, totalizando 30 amostras. Elas foram submetidas à agitação por 30 min com o uso de uma mesa agitadora orbital Solab SL180/D $®$ a $33 \mathrm{rpm}$, e depois foram mantidas em repouso, abrigadas da luz. As alíquotas foram amostradas em intervalos de 1, 2, 4, 8 e 24 horas. Antes das leituras, os frascos foram centrifugados a $1500 \mathrm{rpm}$ por $10 \mathrm{~min}$.

Foram feitas as medições de concentração de uranina nas soluções para a elaboração das curvas de equilíbrio S/Co versus t para cada sedimento.

\section{iii) Variação de $\mathrm{pH}$}

O ensaio de variação de $\mathrm{pH}$ foi elaborado para aferir o comportamento fluorescente da uranina em função da variação do

pH da solução.

A calibração do pHmetro foi feita com soluções tampão de $\mathrm{pH}$ $4,00( \pm 0,02)$ e $7,00( \pm 0,02)$. As medições foram realizadas com um nível de confiança de $96,4 \%$.
Em frascos âmbar, foram montadas soluções de $100 \mathrm{~mL}$ de uranina em água deionizada, sendo 10 para cada concentração, em duplicatas: 10, 75 e $150 \mu \mathrm{g} / \mathrm{L}$, totalizando 60 soluções. Cada amostra foi induzida a um valor de pH entre 3 e 12 por meio do gotejamento de soluções diluídas (10:1) de ácido clorídrico $(\mathrm{HCl})$ e hidróxido de sódio $(\mathrm{NaOH})$ em concentrações de $1 \mathrm{~mol} / \mathrm{L}$.

Para atingir o valor de $\mathrm{pH}$ mais ácido $(\mathrm{pH} 3)$ e os valores mais alcalino ( $\mathrm{pH} 11$ e 12), foram utilizadas soluções não diluídas de $\mathrm{HCl}$ e $\mathrm{NaOH}$ (1 mol/L) respectivamente, para que o volume utilizado do ácido ou da base não ultrapassasse $5 \%$ do volume total da solução contendo uranina.

\subsection{Batch-tests}

Os batch-tests foram realizados em triplicatas, para os três tipos de sedimento, em soluções com água deionizada e água do aquífero, separadamente, na razão de $10 \mathrm{~g}$ de sedimento para $100 \mathrm{~mL}$ de solução, com medições de branco no início e no término dos ensaios. Foram utilizadas as seguintes concentrações iniciais de uranina, em $\mu \mathrm{g} / \mathrm{L}: 5,10,50,100$ e 150 (Figura 2), totalizando 90 amostras. Esse intervalo engloba os valores esperados de concentração em ensaios com uranina em aquíferos ou corpos hídricos superficiais sem que haja riscos tóxico e ecotoxicológicos. Figura 2 - Tipos de solventes, sedimentos e concentrações de uranina
utilizadas nos batch-tests.

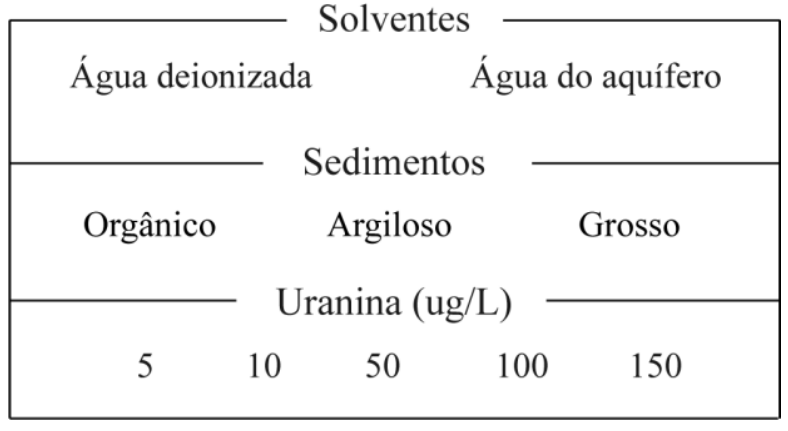

A fluorescência de fundo da água do aquífero e dos sedimentos foi definida através da análise dos brancos. Após agitação por $30 \mathrm{~min}$ a $33 \mathrm{rpm}$ em mesa agitadora orbital Solab SL180/D $\AA$, as amostras foram mantidas em repouso por um tempo de equilíbrio (teq), quantificado através de ensaios de equilíbrio químico: 2 horas para os sedimentos finos e 24 horas para o sedimento grosso. Os frascos foram centrifugados em uma centrífuga de mesa Hermle Z400® a 1500 rpm por $10 \mathrm{~min}$.

\section{RESULTADOS}

\subsection{Tempo de equilíbrio e agitação}

As curvas de adsorção da uranina em relação aos três sedimentos avaliados ao longo de 24 horas estão representadas na Figura 3A. As três curvas atingiram patamares a diferentes concentrações.

Para o sedimento grosso, o equilíbrio foi atingido com cerca de $20 \%$ de uranina adsorvida em aproximadamente 8 horas de repouso. As soluções contendo sedimento orgânico e argiloso entraram em equilíbrio na primeira hora, com aproximadamente $90 \%$ e $35 \%$ de uranina adsorvida, respectivamente. Desta forma, foram obtidos os intervalos de descanso das soluções durante os batch-tests para cada tipo de sedimento, tendo em vista o tempo de equilíbrio químico entre o adsorvato e o adsorvente.

A Figura 3B apresenta a variação da concentração final da uranina na solução em função do tempo de agitação das amos- 
tras contendo os sedimentos avaliados. Não foi observada uma influência significativa do tempo de agitação das soluções na adsorção da uranina para nenhum dos três tipos de sedimento. A maior variação foi constatada para o sedimento argiloso, cuja diferença entre o valor máximo e o valor mínimo foi de $3,9 \mu \mathrm{g} / \mathrm{L}$, observada respectivamente para o tempo mínimo (30 min) e tempo máximo (240 min).
Para o sedimento grosso, a variação entre os valores máximo e mínimo foi de 3,1 $\mu \mathrm{g} / \mathrm{L}$, contudo essa diferença não é linearmente crescente em relação ao tempo de agitação. A concentração final de uranina na solução contendo o sedimento orgânico manteve-se praticamente constante para os três intervalos de agitação.

Figura 3 - (A) Curvas de equilíbrio da uranina para os três sedimentos; (B) Concentração da uranina em função do tempo de agitação para os três sedimentos

Sedimentos

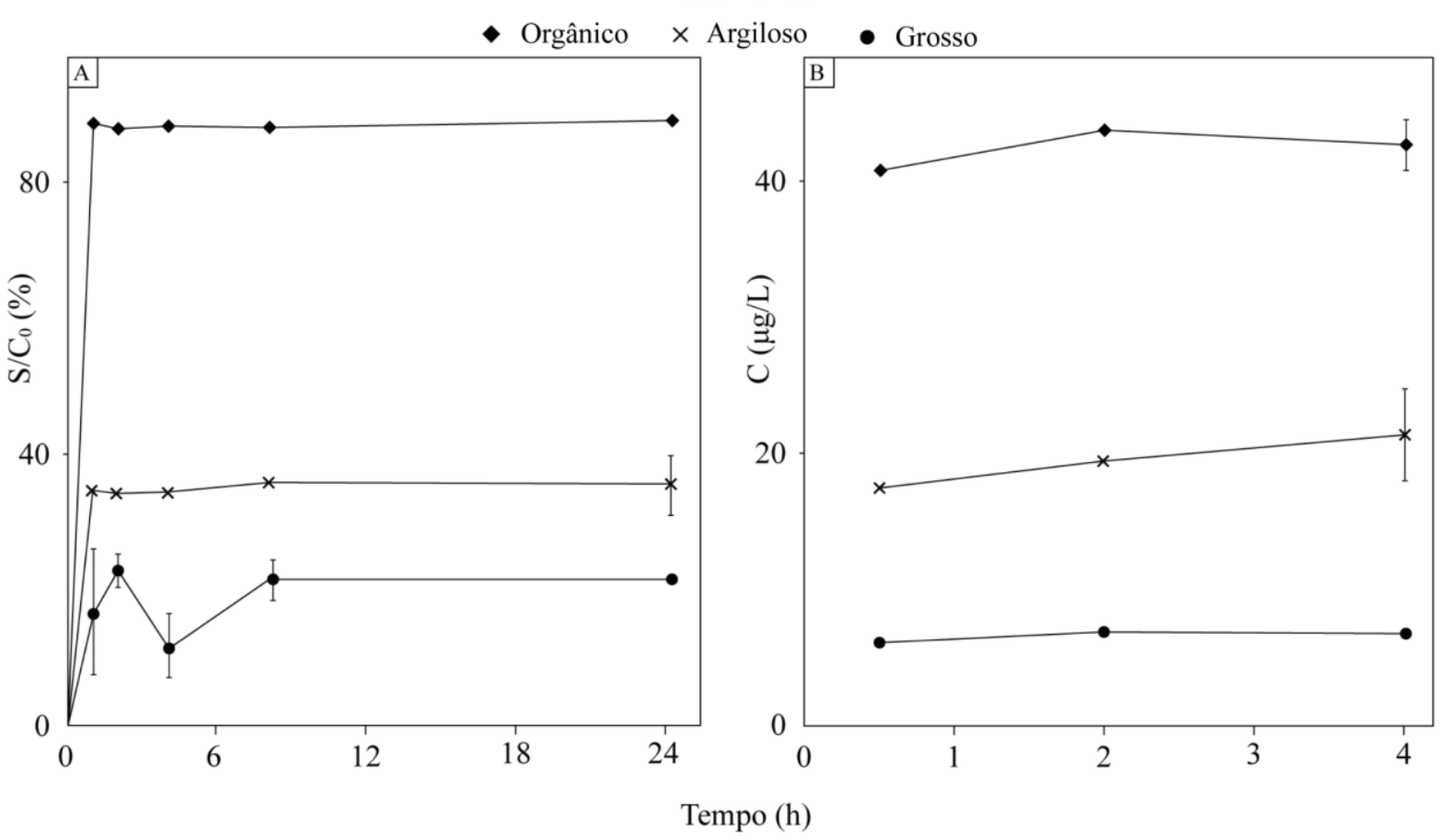

\subsection{Variação de $\mathrm{pH}$}

Observou-se que a propriedade fluorescente das moléculas de uranina está diretamente relacionada ao $\mathrm{pH}$ da solução ( $\mathrm{Fi}$ gura 4). Em soluções de pH ácido, os valores de concentração final não ultrapassaram $50 \%$ da concentração inicial do traçador nas soluções, tendendo a zero com o incremento da acidez.

Para valores de $\mathrm{pH}$ entre 3 e 7 , as curvas $\mathrm{C} / \mathrm{C}_{0}$ versus $\mathrm{pH}$ apre- sentaram um crescimento exponencial em função do aumento do grupo funcional hidroxila nas soluções para as três

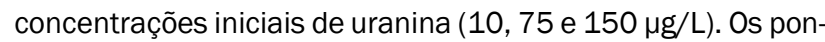
tos de inflexão das curvas ocorrem em pHs levemente alcalinos, já com valores de $\mathrm{C}$ entre $70 \%$ e $90 \%$ em relação a $C_{0}$.

A partir de $\mathrm{pH} 9$, as curvas atingem um patamar e se estabilizam com a razão $\mathrm{C} / \mathrm{C}_{0}$ próxima a 1 , quando a uranina exibe sua máxima intensidade de fluorescência. 


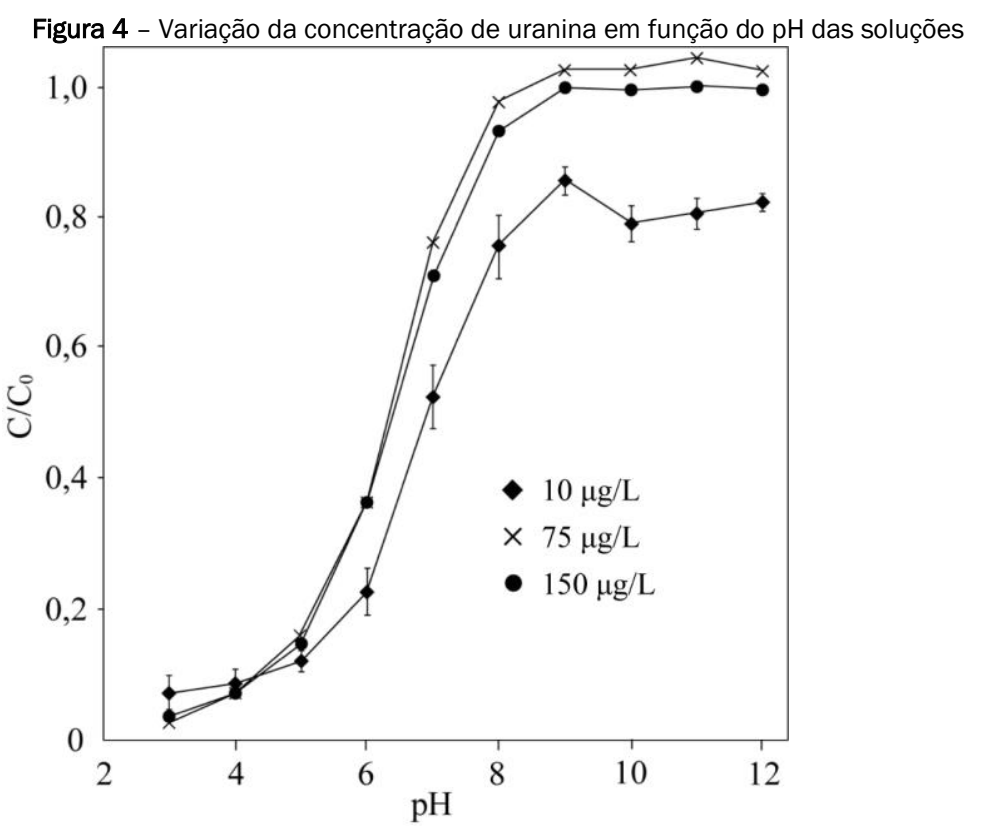

\subsection{Adsorção de uranina nos sedimentos}

Os resultados dos batch-tests realizados a partir da combinação de soluções de água deionizada e do aquífero com distintas concentrações de uranina para os três tipos de sedimento são apresentados a seguir.

\section{i) Sedimentos finos}

Os diagramas apresentados na Figura 5 indicam, para ambos os sedimentos finos, uma correlação não esperada entre a concentração adsorvida (S) de uranina nos sedimentos e a concentração final (C) do traçador. A distribuição dos pontos nos diagramas de dispersão $S$ versus $C$ mostram um alinhamento subvertical, cujas variações dos valores de $C$ são baixas.

As medições obtidas de C para o sedimento orgânico, tanto com a água deionizada quanto com a água do aquífero (Figura 5A e B), para as cinco concentrações iniciais $\left(\mathrm{C}_{0}\right)$ de traçador na solução, variaram de 8,1 $\mu \mathrm{g} / \mathrm{L}$ a $10,2 \mu \mathrm{g} / \mathrm{L}$.

Os valores de concentração dos brancos iniciais (BOi) e finais (BOf) com sedimento orgânico, respectivamente para água deionizada e água do aquífero, foram $\mathrm{BO}$ =
$8,2 \mu \mathrm{g} / \mathrm{L}$ e $9,5 \mu \mathrm{g} / \mathrm{L}$, e BOf $=8,2 \mu \mathrm{g} / \mathrm{L}$ e $7,8 \mu \mathrm{g} / \mathrm{L}$. Ainda para o sedimento orgânico, a concentração adsorvida do traçador variou entre $-3,3 \times 10^{-2} \mathrm{\mu g} / \mathrm{g}$ e $1,42 \mathrm{\mu g} / \mathrm{g}$, nos testes com água deionizada, e entre $-4,3 \times 10^{-2} \mu \mathrm{g} / \mathrm{g}$ e $1,41 \mu \mathrm{g} / \mathrm{g}$ com água do aquífero. Os valores negativos de medição indicam que as amostras apresentaram concentrações levemente inferiores em relação aos brancos utilizados na calibração do fluorímetro. Contudo, esses valores estão dentro da margem de erro de leitura do equipamento.

Os brancos iniciais (BAi) e finais (BAf) para o sedimento argiloso, com as águas deionizada e do aquífero apresentaram resultados semelhantes, com valores de $C$ próximos a $40 \mu \mathrm{g} / \mathrm{L}$. As concentrações finais do traçador nas soluções com água deionizada e sedimento argiloso (Figura $5 \mathrm{C}$ ) variaram de 40,4 $\mu \mathrm{g} / \mathrm{L}$ a $47,4 \mu \mathrm{g} / \mathrm{L}$. Nas soluções contendo a água do aquífero (Figura 5D), as variações de concentração foram muito afins em relação às soluções contendo água deionizada, com concentrações finais oscilando entre $43,9 \mu \mathrm{g} / \mathrm{L}$ e $42,9 \mu \mathrm{g} / \mathrm{L}$, para $\mathrm{C}_{0}$ entre $5 \mu \mathrm{g} / \mathrm{L}$ e $150 \mu \mathrm{g} / \mathrm{L}$. A concentração adsorvida de uranina no sedimento argiloso em função da variação de $C_{0}$ foi semelhante para ambas as águas, com valores variando entre $-3,9 \times 10^{-1} \mu \mathrm{g} / \mathrm{g}$ e $1,0 \mathrm{\mu g} / \mathrm{g}$. 
Figura 5 - Concentração adsorvida versus concentração final de uranina nas soluções com (A) Sedimento orgânico e água deionizada; (B) Sedimento orgânico e água do aquífero; (C) Sedimento argiloso e água deionizada; (D) Sedimento argiloso e água do aquífero

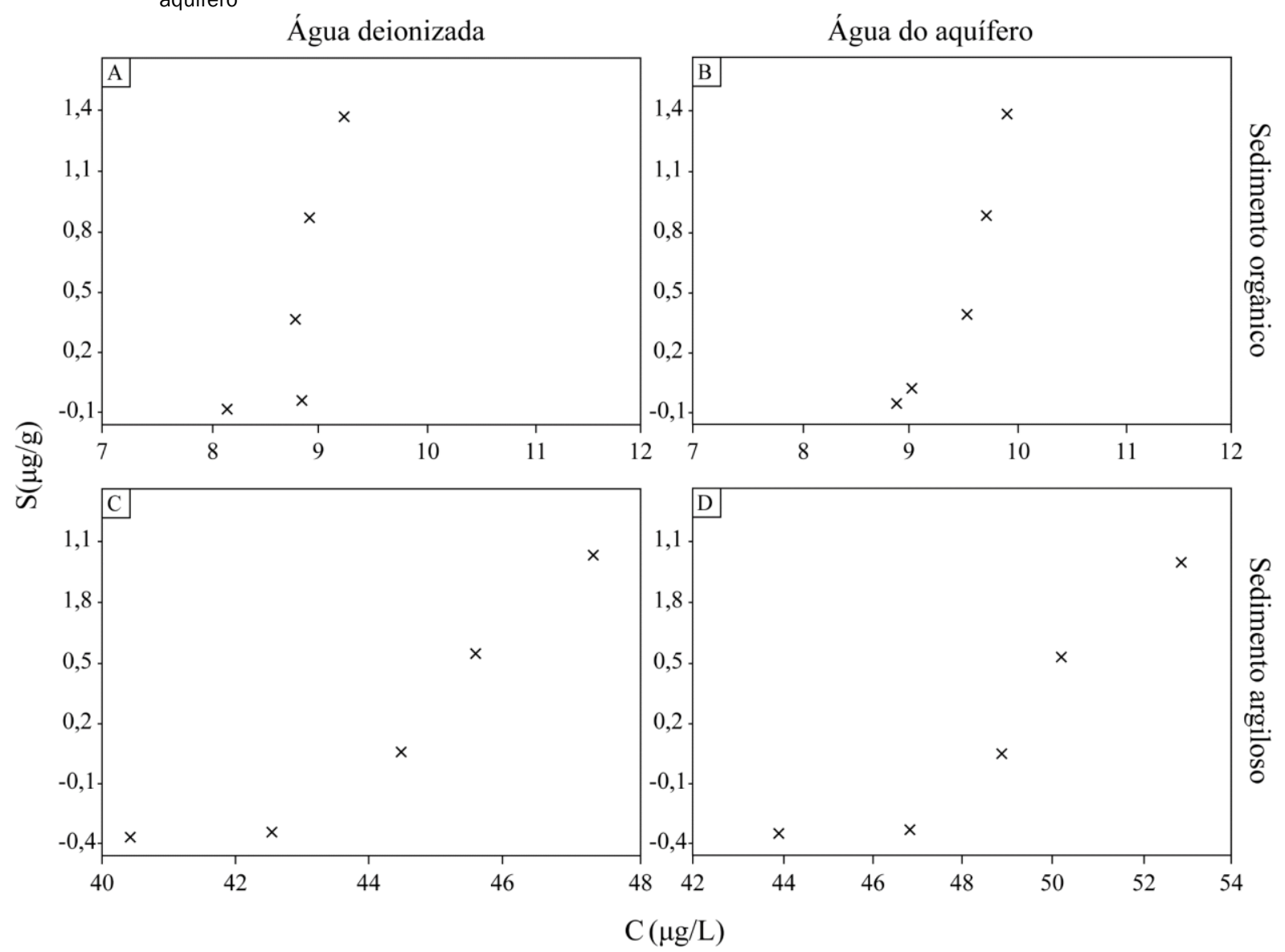

\section{ii) Sedimento grosso}

Os resultados dos batch-tests para o sedimento grosso

A medição dos brancos iniciais (BGi) e finais (BGf) com definiram uma distribuição de tendência linear com expressiva variação de $\mathrm{C}$ nos diagramas de dispersão $\mathrm{S}$ sedimento grosso foram: $\mathrm{BGi}=3,1 \mu \mathrm{g} / \mathrm{L}$ e $0,7 \mu \mathrm{g} / \mathrm{L}$; BGf $=4,3 \mu \mathrm{g} / \mathrm{L}$ e $1,8 \mu \mathrm{g} / \mathrm{L}$, referentes às águas deionizada $\mathrm{e}$ do aquífero, respectivamente.

Figura 6 - Concentração adsorvida versus concentração final de uranina nas soluções com sedimento grosso em (A) água deionizada e (B) água do aquífero com retas ajustadas à distribuição dos pontos Água deionizada Água do aquífero

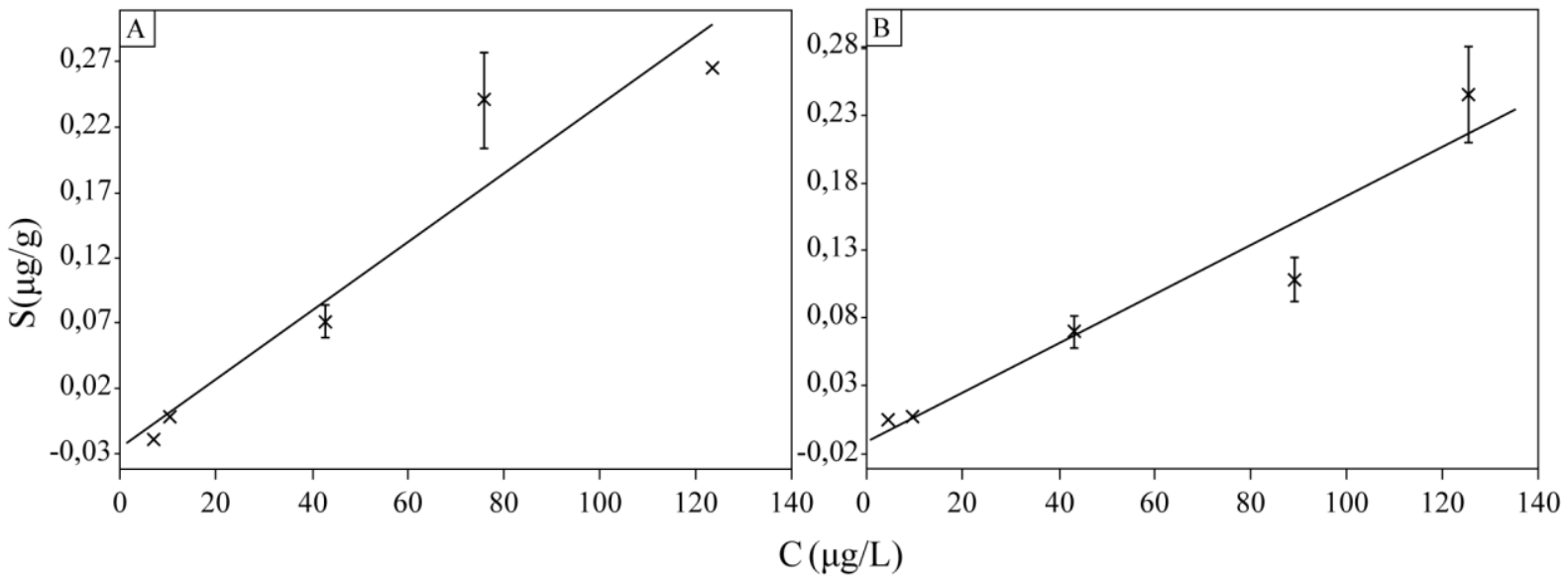


Para os ensaios realizados com água deionizada (Figura $6 \mathrm{~A})$, os valores obtidos de $\mathrm{S}$ variaram de $-1,90 \times 10^{-2} \mathrm{\mu g} / \mathrm{g}$

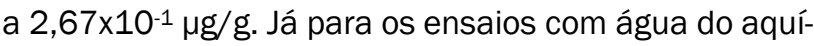
fero (Figura 6B), a concentração adsorvida do traçador no sedimento variou entre $4,00 \times 10^{-3} \mathrm{\mu g} / \mathrm{g}$ e $2,88 \times 10^{-1}$, respectivamente para $\mathrm{C}_{0 m i ́ n}$ e Comáx.

As concentrações finais de uranina nas soluções, para os cinco valores de $\mathrm{C}_{0}$, atingiram valores muito próximos para as duas águas, variando entre 4,4 $\mu \mathrm{g} / \mathrm{L}$ e 127,8 $\mu \mathrm{g} / \mathrm{L}$.

\subsection{Definição do fator de retardamento}

A determinação do fator de retardamento $(\mathrm{R})$ para o sedimento grosso foi baseada no coeficiente de partição $\left(K_{d}\right)$ obtido através do ajuste de isotermas lineares nos diagramas $S$ versus $C$. Os ajustes das funções afins (Figura $6 \mathrm{~A}$ e $6 \mathrm{~B}$ ) foram obtidos pelo método dos mínimos quadrados lineares com coeficientes de determinação superiores a 0,9.

Os valores de porosidade específica $\left(n_{e}\right)$ e densidade seca dos sedimentos $\left(\rho_{b}\right)$, utilizados na estimativa do fator de retardamento, foram: $\mathrm{n}_{\mathrm{e}}=9,2 \%$ e $\rho_{\mathrm{b}}=1,79 \mathrm{~g} / \mathrm{cm}^{3}$ (Tabela 3).

Tabela 3 - Valores de porosidade específica, densidade seca, coeficiente de adsorção e fator de retardamento para o sedimento grosso para as duas águas utilizadas

\begin{tabular}{llllll}
\hline Sedimento & Água & $\begin{array}{l}\mathrm{n}_{\mathrm{e}} \\
(\%)\end{array}$ & $\begin{array}{l}\mathrm{\rho b}_{\mathrm{b}} \\
\left(\mathrm{g} / \mathrm{cm}^{3}\right)\end{array}$ & $\begin{array}{l}\mathrm{Kd} \\
\left(10^{-3} \mathrm{~L} / \mathrm{g}\right)\end{array}$ & $\begin{array}{l}\text { Fator de } \\
\text { retardamento }\end{array}$ \\
\hline \multirow{2}{*}{ Grosso } & Deionizada & & & $2,60 \pm 0,22$ & $1,51 \pm 0,13$ \\
& & 9,2 & 1,79 & $1,80 \pm 0,12$ & $1,35 \pm 0,09$ \\
\hline
\end{tabular}

Os valores calculados dos coeficientes de adsorção e dos fatores de retardamento, respectivamente, foram $\mathrm{K}_{\mathrm{d}}=2,60 \times 10^{-3} \mathrm{~L} / \mathrm{g} \pm 0,22 \times 10^{-3} \mathrm{~L} / \mathrm{g}$ e $\mathrm{R}=1,51 \pm 0,13$ (água deionizada), e $\mathrm{K}_{d}=1,80 \times 10^{-3} \mathrm{~L} / \mathrm{g} \pm 0,12 \times 10^{-3} \mathrm{~L} / \mathrm{g}$ e $\mathrm{R}=1,35 \pm 0,09$ (água do aquífero).

\section{DISCUSSÃO}

O ensaio de variação de $\mathrm{pH}$ das amostras contendo 10, 75 e $150 \mu \mathrm{g} / \mathrm{L}$ de uranina apresentou resultados que confirmam a forte correlação entre a intensidade de flu- orescência do traçador e o grau de acidez das soluções. Os resultados obtidos estão de acordo com as referências literárias (Figura 7), conforme Kass (1998). A dissimilaridade do comportamento da curva para $\mathrm{C}_{0}=10$ $\mu \mathrm{g} / \mathrm{L}$ em relação às curvas para $\mathrm{C}_{0}=75 \mu \mathrm{g} / \mathrm{L}$ e $\mathrm{C}_{0}=150$ $\mu \mathrm{g} / \mathrm{L}$, na Figura 4, pode ser entendida através da maior sensibilidade a erros associados ao método (diluição, variação de temperatura, titulação e leitura) para concentrações iniciais mais baixas.

Figura 7 - Variação da intensidade de fluorescência da uranina em função

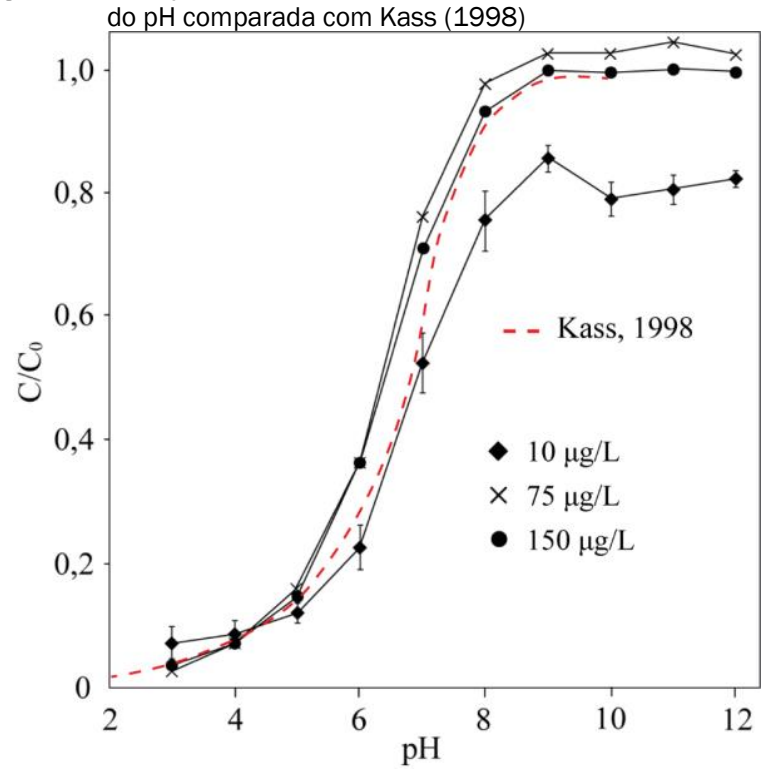


Para maximizar o tempo de trabalho em análises laboratoriais do comportamento sorptivo da uranina, podese adotar intervalos de repouso próximos a 8 horas com períodos de agitação inferiores a 4 horas, desde que a proporção entre solvente (água), adsorvente (sedimento) e adsorvato (uranina) nas soluções mantenhase dentro dos intervalos utilizados neste estudo.

A influência do grau de cristalinidade da água nas leituras realizadas pelo fluorímetro pôde ser constatada através das medições dos brancos (I) contendo apenas as águas utilizadas nos experimentos e dos brancos (II) compreendendo sedimento + água. 0 tempo e a intensidade do processo de centrifugação não foram suficientes para a decantação total dos sedimentos em suspensão, gerando medições em soluções moderadamente turvas.

Em (I), as concentrações obtidas variaram entre -1,8 $\mu \mathrm{g} / \mathrm{L}$ e 0,9 $\mu \mathrm{g} / \mathrm{L}$, denotando caráter não fluorescente de ambas as águas para os espectros de excitação e emissão trabalhados. As medições em (II) indicaram valores semelhantes às concentrações finais obtidas durante os batch-tests para as cinco concentrações iniciais utilizadas, tanto para o sedimento orgânico quanto para o argiloso.

Dessa forma, os batch-tests realizados para os sedimentos finos não apresentaram os resultados esperados, uma vez que a fluorescência aparente provocada pela presença de partículas em suspensão nessas soluções se sobrepôs à fluorescência da uranina dissolvida. Suhogusoff et al. (2005), por meio de ensaios com a substância fluorescente uranina, definiram para os sedimentos arenosos da Bacia de São Paulo um valor de $\mathrm{K}_{d}$ próximo aos obtidos neste trabalho $\left(3,72 \times 10^{-3} \mathrm{~L} / \mathrm{g} \pm\right.$ $\left.0,01 \times 10^{-3} \mathrm{~L} / \mathrm{g}\right)$. Experimentos com uranina realizados por Sabatini e Austin (1991) e Smart e Laidlaw (1977) indicam que o traçador sofre com altas taxas de adsorção em matéria orgânica e argilominerais do grupo da caulinita em suspensão. Contudo, em sedimentos arenosos, de composição majoritariamente quartzosa, esse comportamento não foi observado.

\section{CONCLUSÕES}

Os resultados obtidos neste trabalho apontam que a intensidade de fluorescência da uranina apresenta forte dependência em relação às variações de $\mathrm{pH}$ das soluções, atingindo o valor máximo em meio alcalino superior ao pH 9. Desta forma, recomenda-se a realização de ajuste no pH (alcalinização) de soluções contendo uranina antes da quantificação de sua fluorescência.
A fluorescência de fundo gerada por matéria orgânica coloidal e argilominerais em suspensão se sobrepôs à fluorescência real do traçador, mesmo após a decantação da maior parte desse material através de centrifugação.

A uranina não se comporta como um traçador artificial ideal em meios sólidos que apresentam alto conteúdo de matéria orgânica e argilominerais do grupo da caulinita.

Espera-se, também, que a uranina apresente uma maior susceptibilidade aos fenômenos de sorção em argilominerais cuja capacidade de troca catiônica (CTC) seja superior à CTC observada na caulinita (e.g. montmorilonita e vermiculita).

Em meios francamente quartzosos, com contribuição discreta de matéria orgânica e argilominerais, é possível utilizar a uranina como uma ferramenta de aferição das propriedades hidráulicas de um aquífero, uma vez que ela apresenta um comportamento pouco sorptivo e, portanto, um baixo fator de retardamento.

\section{REFERÊNCIAS}

BORK, M.; LANGE, J.; GRAF-ROSENFELLNER, M.; LANG, F. Controls of fluorescent tracer retention by soils and sediments. Hydrology and Earth System Sciences, 24, p. 977-989, 2020. https://doi.org/10.5194/hess-24977-2020

BOTTRELL, S. H.; THORNTON, S. F.; SPENCE, M. J.; ALLSHORN, S.; SPENCE, K. H. Assessment of the use of fluorescent tracers in a contaminated Chalk aquifer. Quarterly Journal of Engineering Geology and Hydrogeology, v. 43, n. 2, p. 195-206, 2010.

https://doi.org/10.1144/1470-9236/08-020

CAO, V.; SCHAFFER, M.; TAHERDANGKOO, R.; LICHA, T. Solute Reactive Tracers for Hydrogeological Applications: A Short Review and Future Prospects. Water. v. 12, n. 3, p. 653, 2020.

https://doi.org/10.3390/w12030653

DIVINE, C.E.; MCDONNELL, J.J. The future of applied tracers in hydrogeology. Hydrogeology Journal, 13, p. 255-258, 2005. https://doi.org/10.1007/s10040$\underline{\text { 004-0416-3 }}$

FERNANDES, A.J.; PERROTTA, M.M.; SALVADOR, E.D.; AZEVEDO, S.G.; GIMENEZ FILHO, A.; PAULON, N. Potencial dos aquíferos fraturados do Estado de São Paulo: condicionantes geológicos. Revista Águas Subterrâneas. $\quad$ v. 21, p. 63-84, 2007. https://doi.org/10.14295/ras.v21i1.16168 
FIELD, M.S.; WILHELM, R.G.; QUINLAN, J.F.; ALEY, T.J. An assessment of the potential adverse properties of fluorescent tracer dyes used for groundwater tracing. Environ. Monit. Assess, v. 38, p. 75-97, 1995. https://doi.org/10.1007/BF00547128

GOMBERT, P.; BIAUDET, H.; DE SEZE, R.; PANDARD, P.; CARRÉ, J. Toxicity of fluorescent tracers and their degradation byproducts. International Journal of Speleology. V. 46, p. 1, p. 23-31, 2017. https://doi.org/10.5038/1827-806X.46.1.1995

HASUI, Y.; CARNEIRO, C.D.R.; COIMBRA, A.M. The Ribeira Folded Belt. Revista Brasileira de Geociências, v. 5, n. 4, p. 257-266, 1975. https://doi.org/10.25249/03757536.1975257266

INSTITUTO DE PESQUISAS TECNOLÓGICAS DO ESTADO DE SÃO PAULO - IPT. Investigação detalhada, avaliação de risco à saúde humana e plano de intervenção - área da UTM-Jaguaré, no bairro Jaguaré, São Paulo-SP. 2017.

JANASI, V.A.; ULBRICH, H.H.G.J. Late Proterozoic granitoid magmatism in the state of São Paulo, southeastern Brazil. Precambrian Research, v. 51, n. 1, p. 351- 374, 1991.https://doi.org/10.1016/0301-9268(91)90108$\underline{\mathrm{M}}$

JULIANI, C. Geologia, petrogênese e aspectos metalogenéticos dos grupos Serra do Itaberaba e São Roque na região das Serras do Itaberaba e da Pedra Branca, NE da Cidade de São Paulo, SP. Tese (Doutorado) - Instituto de Geociências, Universidade de São Paulo, 1993.

KASS, W. Geohydrologische markierungstechnik. Gebruder Bornstraeger. Berlin/ Stuttgart, 1998. 581 p.

LEIBUNDGUT, C.; MALOSZEWSKI, P.; KÜLLS, C. Tracers in Hydrology. John Wiley e Sons, Ltd. 2009. https://doi.org/10.1002/9780470747148

LEIBUNDGUT, C.; SEIBERT, J. Tracer Hydrology Volume 2: the science of hydrology. Elsevier Science. p. 215236, 2011. https://doi.org/10.1016/B978-0-44453199-5.00036-1

PONSIN, V.; CHABLAIS, A.; DUMONT, J.; RADAKOVITCH, O.; E HÖHENER, P. 222 Rn as Natural Tracer for LNAPL Recovery in a Crude Oil-Contaminated Aquifer. Groundwater Monitoring e Remediation, v. 35, n. 2, p. 30-38, 2015. https://doi.org/10.1111/gwmr.12091

RIBEIRO, C.B.M. Sistema de alerta ambiental fundamentado em estudo teórico-experimental de transporte e dispersão de poluentes solúveis em cursos d'água. Tese (Doutorado) - Universidade Federal de Viçosa, Minas Gerais, Brasil, 2007.
RICCOMINI, C. O Rift continental do sudeste do Brasil. Tese (Doutorado) - Instituto de Geociências, Universidade de São Paulo, São Paulo, Brasil. 1990.

RICCOMINI, C.; SANT'ANNA, L.G.; FERRARI, A.L. Evolução geológica do rift continental do sudeste do Brasil. In: Geologia do continente Sul-Americano: evolução da obra de Fernando Flávio Marques de Almeida. p. 383-405, 2004.

ROCHA, G.A. Mapa de águas subterrâneas do Estado de São Paulo. Escala 1:1.000.000. Nota explicativa. São Paulo. DAEE-Departamento de Águas e Energia Elétrica; IG- Instituto Geológico; IPT-Instituto de Pesquisas Tecnológicas; CPRM-Serviço Geológico do Brasil, 2005.

RODRIGUEZ, S.K. Geologia urbana da região metropolitana de São Paulo. Tese (Doutorado) - Instituto de Geociências, Universidade de São Paulo, São Paulo, Brasil, 1998.

SABATINI, D. A.; AUSTIN, T. Characteristics of rhodamine WT and fluorescein as adsorbing ground-water tracers. Groundwater, v. 29, n. 3, p. 341-349, 1991. https://doi.org/10.1111/i.1745-584.1991.tb00524.x

SILVA, L.L.; DONNICI, C.L.; AYALA, J.D.; FREITAS, C.H.; MOREIRA, R.M.; PINTO, A.M.F. Traçadores: o uso de agentes químicos para estudos hidrológicos, ambientais, petroquímicos e biológicos. Química Nova. v. 32, n. 6, p. 1576-1585, 2009. https://doi.org/10.1590/S010040422009000600042

SMART, P.L.; LAIDLAW, I.M.S. An evaluation of some fluorescent dyes for water tracing. Water Resources Rese$\begin{array}{lllll}\text { arch, } & \text { v. 13, p. 15-32, } 1977 .\end{array}$ https://doi.org/10.1029/WR013i001p00015

SUHOGUSOFF, A. V.; HIRATA, R.; FERRARI L. C. K. M. Adsorção do traçador fluorescente uranina em sedimentos quaternários da Bacia de São Paulo. Revista Brasileira de Geociências, v. 35, p. 551-558, 2005. https://doi.org/10.25249/0375-536.200537551558

TAKIYA, H. Estudo da sedimentação Neogênico-Quaternário no município de São Paulo: caracterização dos depósitos e suas implicações na geologia urbana. Tese (Doutorado) - Instituto de Geociências, Universidade de São Paulo, São Paulo, Brasil, 1997.

TRUDGILL, S.T. Soil water dye tracing with special reference to the use of rhodamine WT, lissamine FF, and amino G acid. Hydrological Processes. 1, 149-170, 1987. https://doi.org/10.1002/hyp.3360010204 
TURNER DESIGNS. TD-700. Laboratory Fluorometer Operating Manual. 2002. Disponível em: http://docs.turnerdesigns.com/t2/doc/manuals/7000-998.pdf. Acesso em: 16 jan. 2021.

WILSON JR, J. F.; COBB, E. D.; KILPATRICK, F. A. Techniques of water-resourses investigations of the United States Geological Survey. Fluorometric Procedures for Dye Tracing. Book 3 - Applications of Hydraulics. U. S. Geological Survey. 34 p, 1986.
ZHENG, C. MT3D: a modular three-dimensional transport model for simulation of advection, dispersion and chemical reactions of contaminants in groundwater systems. The United States Environmental Protection Agency. Ada, Oklahoma.163p, 1990. 